\title{
Synthesis of novel metal containing epoxy polymers and their structural characterization by means of FT-IR and coupled TG/MS measurements
}

Ivan Ristic ${ }^{1 *}$, Berta Barta Holló ${ }^{2}$, Jaroslava Budinski-Simendić ${ }^{1}$, Katalin Mészáros Szécsényi ${ }^{2}$, Suzana Cakić $^{3}$, Imre Miklós Szilágyi ${ }^{4,5}$, György Pokol ${ }^{5}$

${ }^{1}$ University of Novi Sad, Faculty of Technology, Novi Sad, Serbia

${ }^{2}$ University of Novi Sad, Faculty of Science, Serbia

${ }^{3}$ University of Nis, Faculty of Technology, Leskovac, Serbia

${ }^{4}$ MTA-BME Technical Analytical Chemistry Research Group, H-1111 Budapest, Szent Gellért tér 4., Hungary

${ }^{5}$ Department of Inorganic and Analytical Chemistry, Budapest University of Technology and Economics, H-1111 Budapest, Szent Gellért tér 4., Hungary

\begin{abstract}
Novel copper containing epoxy polymers have been synthesized using copper(II) formamidine complex $\left[\mathrm{Cu}(\operatorname{ampf}) \mathrm{Cl}_{2}\right]$, ampf $=N, N^{\prime}$-bis(4-acetyl-5-methylpyrazole-3-yl)formamidine, as curing agent for diglycidyl ether of bisphenol A based epoxy resins (Araldite and its oligomer consisting of 3-4 monomer units). The curing reaction was performed at room temperature without catalyst. In order to prove the polymerization reaction, differential scanning calorimetry (DSC) was applied. Highly exothermic peaks around $150{ }^{\circ} \mathrm{C}$ refer to the polymerization and according to DSC results the epoxy resins above $200{ }^{\circ} \mathrm{C}$ were almost completely converted to the corresponding metal-containing polymer. The proposed structure of the hybrid polymers was confirmed by FT-IR analysis. Gel permeation chromatography analysis shows that the average molar masses of the polymers are in the range from 3000 to $50000 \mathrm{~g} \mathrm{~mol}^{-1}$. The low conductivities of the hybrid materials in tetrahydrofuran confirm the non-ionic structure of the new polymers in solution. The thermal stability and the decomposition mechanism of the components of the reaction mixture and the new polymers were studied by coupled TG/DTA-MS measurements. The thermal data were analyzed also with the aim to obtain additional information about the composition of the synthesized materials.
\end{abstract}

Key words: epoxy, metal containing polymers, FT-IR, thermal properties, TG/MS

"Corresponding author: Ivan S. Ristić, University of Novi Sad, Faculty of Technology, Bulevar cara Lazara 1, 21000 Novi Sad, Serbia

e-mail: ivancekaris@yahoo.com

phone: +381214853760

fax: +38121450413 


\section{Introduction}

Epoxy polymers are one of the most important high-performance materials in use today, ranging from simple two-part adhesives and sports equipment to high-tech applications such as electronics devices, racing cars, the aerospace industry etc. The enhanced reactivity towards a variety of reagents makes the epoxy resin a versatile curing agent. Curing agents based on polyamines are among the most commonly used ones in epoxy resin systems and make up around $22 \%$ of the commercial reagents used [1]. Both primary and secondary amines may be used to initiate cure in epoxy systems and are generally employed in stoichiometric amounts. Different classes of metal compounds may be used as cross-linkers of epoxy oligomers: organometallic compounds, salts with inorganic and organic counter-ions, metal complexes [2], etc. As for the latter ones, chelates are predominantly used like $\beta$ diketonates and complexes with oxygen and nitrogen containing ligands, in particular, aliphatic amines [3]. To prepare metal-containing epoxy polymers metal chelates are used as curing agents [46] and organotransition-metal complexes are applied as additives [7-9]. The epoxy resin with arsenic cured with polyamide showed chemical resistance and excellent thermal stability compared with epoxy resin without arsenic [10-12]. For optimization of the curing regime for epoxy-chelate composite materials in order to decrease the residual stresses, transition metal complexes were used as curing agents [13]. Metal-containing epoxy polymers are thermally relatively stable [14], possess high strength and can be used for industrial production of one-plate glass-reinforced plastic springs for large-loaded motor vehicles [15]. To improve the physical properties of polymeric materials (such as adhesion, flexural strength, fracture toughness and heat resistance) organotransition metal complexes have also been added. Their presence can increase the density of the resin or adversely affect the conductivity of the cured resin and therefore restrict the applicability in certain electronic applications [16]. In order to enhance the thermal, physical and mechanical properties of the cured resin, metal ammine complexes have been used. Kurnoskin published several papers dealing with introducing metal ions into an epoxy resin structure using polyamine-based metal complexes [17-19].

In the present work we report the synthesis, the structure and the electric conductivity of new metal containing epoxy polymers obtained by curing Araldite GY 250 and CHS-EPOXY 210 X 75 epoxy resins based on Bisphenol A with copper(II) formamidine $\left[\mathrm{Cu}(\operatorname{ampf}) \mathrm{Cl}_{2}\right]$ complex, where ampf stands for $N, N^{\prime}$-bis(4-acetyl-5-methylpyrazole-3-yl)formamidine ligand. The NH-group of the pyrazole ring and the 4-acetyl substituent of the ligand react with epoxy groups of the resins yielding copper(II) containing epoxy polymers.

\section{Experimental}

\subsection{Materials}

Two types of epoxy resins were used. Araldite GY 250 unmodified medium viscous epoxy resin marked as 1 based on Bisphenol A (epoxy value $5.30-5.46$ eq $\mathrm{kg}^{-1}$, viscosity at $25{ }^{\circ} \mathrm{C} 10-12 \mathrm{~Pa} \mathrm{~s}$ ) was supplied from Jubail Chemical Industries Co. CHS-EPOXY 210 X 75 is a $75 \%$ xylene solution 
of an unmodified low molecular mass epoxy resin, 2 (epoxy index: $2-2.25 \mathrm{~mol} \mathrm{~kg}^{-1}$, viscosity at 25 ${ }^{\circ} \mathrm{C}$ 5-12 Pa s) was supplied from Spolchemie a.s, Česká Republika.

Complex $\left[\mathrm{Cu}(\operatorname{ampf}) \mathrm{Cl}_{2}\right]$, ampf $=N, N^{\prime}$-bis(4-acetyl-5-methylpyrazole-3-yl)formamidine) was synthesised and characterized as was described [20]. In further text it is abbreviated as ML. Hybrid polymer prepared using $\mathbf{1}$ is denoted as I and that based on $\mathbf{2}$ as II.

\subsection{Analytical methods}

The average molecular mass of the new polymers was determined at $25{ }^{\circ} \mathrm{C}$ by gel permeation chromatography (GPC) using Agilent 1100 Series system with refractive index, RID 1200, diode array 1200 detectors, column ZORBAX PSM 300 and tetrahydrofuran as eluent (flow rate: $1 \mathrm{~cm}^{3} \mathrm{~min}^{-}$

${ }^{1}$ ). Poly(styrene) standards were used for calibration curve. The average molecular masses and polydispersity indexes $(Q)$ were determined employing software Agilent ChemStation.

The epoxy hybrid materials were characterised by FT-IR spectrometry (Bomem Hartmann \& Braun MB-series). The data were recorded in 400 to $4000 \mathrm{~cm}^{-1}$ IR range using $\mathrm{KBr}$ pellets $(0.5 \mathrm{mg}$ sample/150 $\mathrm{mg} \mathrm{KBr}$ ). The conductivity of the freshly prepared solutions of hybrid materials in tetrahydrofuran (THF) was measured at room temperature using a digital conductivity meter Jenway 4010. Thermal effects of the polymerisation of epoxy resins with ML were measured using Q20 DSC (TA Instruments, USA) calibrated against indium. Standard aluminium pans with lid containing 3-5 mg of reaction mixture were scanned at a heating rate of $2{ }^{\circ} \mathrm{C} \mathrm{min}^{-1}$ in temperature range from 20 to $230^{\circ} \mathrm{C}$.

Thermogravimetric analysis (TG) was performed by simultaneous TG/DSC thermal analyser SDT Q600 (TA Instruments, USA). Sample $(\sim 1.5 \mathrm{mg})$ was placed in an open alumina pan. The measurements were carried out in nitrogen or air atmosphere (flow rate: $100 \mathrm{~cm}^{3} \mathrm{~min}^{-1}$ ) to $600{ }^{\circ} \mathrm{C}$ with a heating rate of $20^{\circ} \mathrm{C} \mathrm{min}^{-1}$. TG/DTA-MS data were collected using TA Instruments SDT 2960 DTA/TG coupled online with Balzers Instruments ThermoStar GSD 300T quadrupole mass spectrometer at $10{ }^{\circ} \mathrm{C} \mathrm{min}{ }^{-1}$ heating rate in $\mathrm{N}_{2}$ and air atmosphere (flow rate $130 \mathrm{~cm}^{3} \mathrm{~min}^{-1}$ ). Selected ions between $\mathrm{m} / \mathrm{z}=1-190$ were monitored through 64 channels in Multiple Ion Detection Mode (MID) with a measuring time of $0.5 \mathrm{~s}$ per channel.

\subsection{Synthesis}

\subsubsection{Preparation of metal-containing epoxy polymers}

A mixture of epoxy resin and ML in an equimolar ratio (for $\mathbf{I}: 1.0 \mathrm{mg}$ of $\mathbf{1}$ and $1.12 \mathrm{mg}$ of ML; for II: $3.3 \mathrm{mg}$ of $\mathbf{2}$ and $1.49 \mathrm{mg}$ of ML) was added to a glass vessel, degassed under vacuum and cured at room temperature. The curing reaction was followed by the decreasing in intensity of the characteristic band of the epoxy groups at $917 \mathrm{~cm}^{-1}$ in the IR spectrum, and according to the IR results, the reactions were accomplished in a 3 days. Samples obtained in reaction of complex with 
epoxy resin 1 are forest green, highly viscous liquids. Samples with resin $\mathbf{2}$ are hard and translucent of light green colour. The solubility of the new materials in THF is about $2 \mathrm{mg} \mathrm{cm}^{-3}$. The conductivity values of THF solutions are comparable with the conductivity for pure THF $\left(\lambda_{\mathrm{THF}}=0.11 \mathrm{Scm}^{2} \mathrm{~mol}^{-1}\right)$ in accordance with the non-ionic nature of the polymers: $\lambda(\mathbf{I})=0.18, \lambda(\mathbf{I I})=0.14 \mathrm{Scm}^{2} \mathrm{~mol}^{-1}$.

\section{Results and discussion}

Differential scanning calorimetry has extensively used to study the curing of epoxy resins [21,22]. The polymerisation of epoxy resins with the metal complex was studied using non-isothermal DSC method. A stoichiometric mixture of ML to resin mole ratio was heated to $230{ }^{\circ} \mathrm{C}$ with a heating rate of $2{ }^{\circ} \mathrm{C} \min ^{-1}$ using standard aluminum pan with lid against the corresponding reference pan. DSC scan of reaction between ML and 1 shows two exothermic peaks with peak maxima at $122{ }^{\circ} \mathrm{C}$ and at $157^{\circ} \mathrm{C}$ indicating curing (Fig. 1a). As can be seen, the epoxy monomer 1 was almost completely converted to the corresponding metal-containing polymer at $<190{ }^{\circ} \mathrm{C}$. The value of reaction enthalpy is $123 \mathrm{~J} \mathrm{~g}^{-1}$. In the reaction of ML complex and epoxy $\mathbf{2}$ only one exothermic peak appears at $171{ }^{\circ} \mathrm{C}$ and the polymerization enthalpy amounts $104 \mathrm{~J} \mathrm{~g}^{-1}$ which suggests a lower reactivity of epoxy resin 2 with the complex, compared to the resin $\mathbf{1}$.

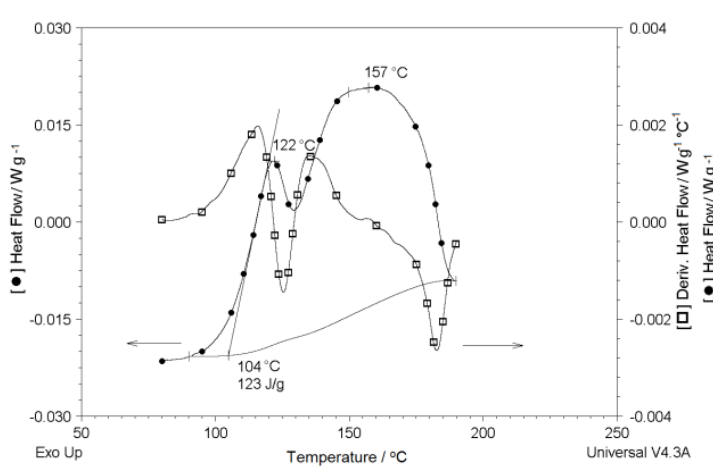

a)

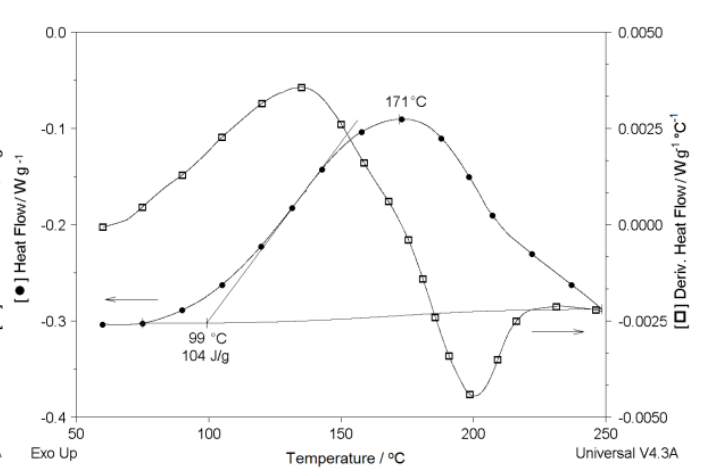

b)

Fig. 1 DSC and derivative DSC curves of polymerization reaction between complex and epoxy resins: a) araldite $\mathbf{1}$, and b) CHS epoxy 2

To accommodate steric strain in the curing reaction, the hydrogen atom from $\mathrm{NH}$-group of the metallocycle migrates to oxygen of the acetyl group which is not coordinated to the metal centre giving thus an $\mathrm{OH}$-group as is presented in Scheme 1. In curing reaction this group and the NH-group of the pyrazole ring react with the oxirane groups of the epoxy resins as is presented in Scheme 2.

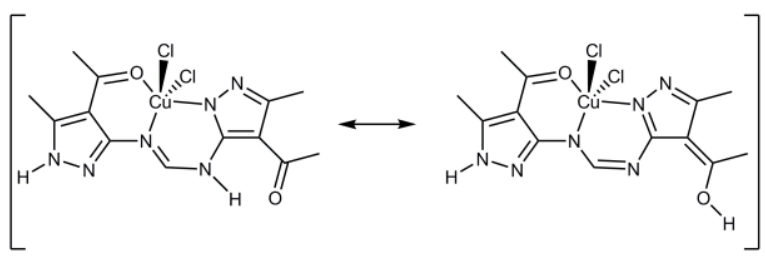

Scheme 1 Tautomeric forms of ML 

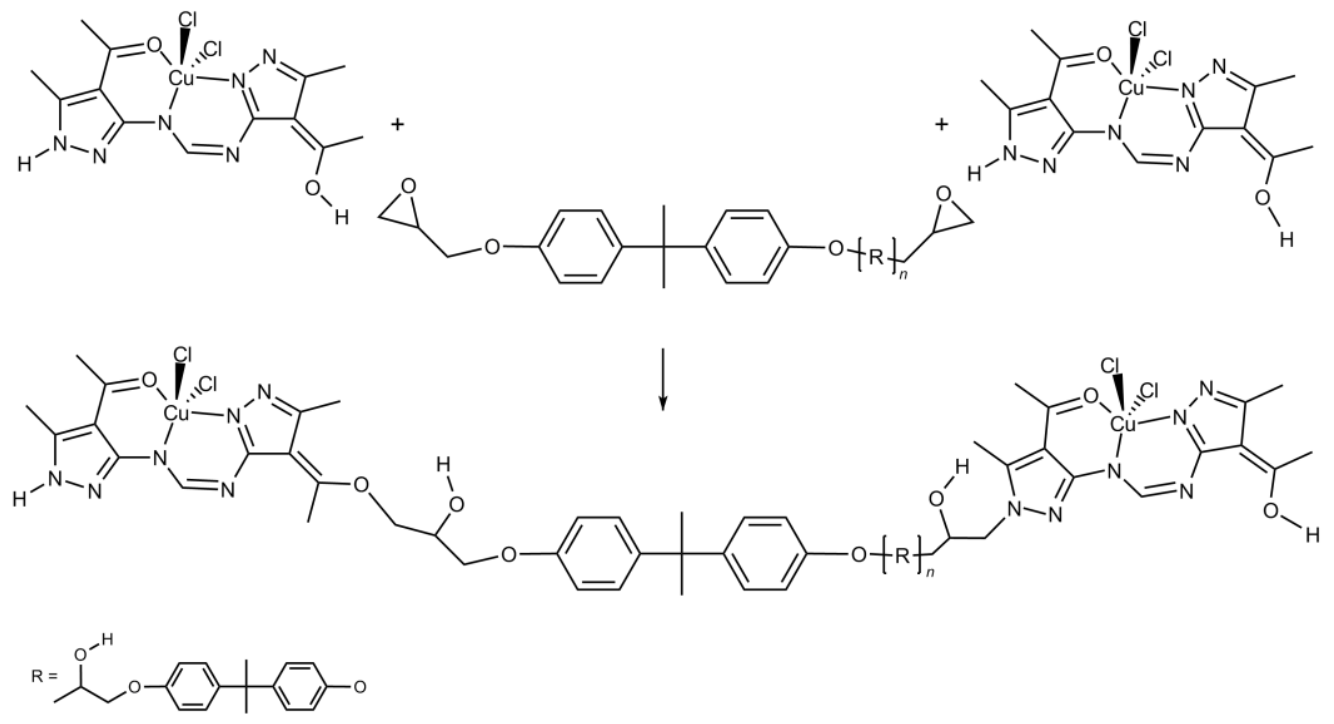

Araldite: $n=0$

CHS-epoxy: $n=3-4$

Scheme 2 Curing reaction of ML and $1(n=0)$ or $\mathbf{2}(n=3-4)$

The molecular masses distribution is relatively broad with polydispersity indexes (Q) ranging from 1.4 to 2.0. The peaks for epoxy resins registered at $5.58 \mathrm{~min}(\mathbf{1})$ and $5.43 \mathrm{~min}$ (2) in GPC chromatograms are missing in the chromatograms of the synthesized hybrids. The chromatograms presented in Figure 2a) and 2 b) clearly show that the hybrid polymer, obtained on the basis of $\mathbf{1}$ have a higher polydispersity index (Q). Molecular masses in $\mathbf{I}$ are in the $25100-49700 \mathrm{~g} \mathrm{~mol}^{-1}$ range $(\mathrm{Q}=$ 1.98), compared with $3200-4480 \mathrm{~g} \mathrm{~mol}^{-1}(\mathrm{Q}=1.40$ ) ones in the corresponding hybrid II, based on oligo-epoxy 2. Also, GPC chromatogram of II based on 2 shows a higher peak multiplicity originating from the polymers with different molecular masses. The higher number of peaks in GPC curves of II is expected as a result of the higher steric hindrances during polymerisation of $\mathbf{2}$ with ML. 


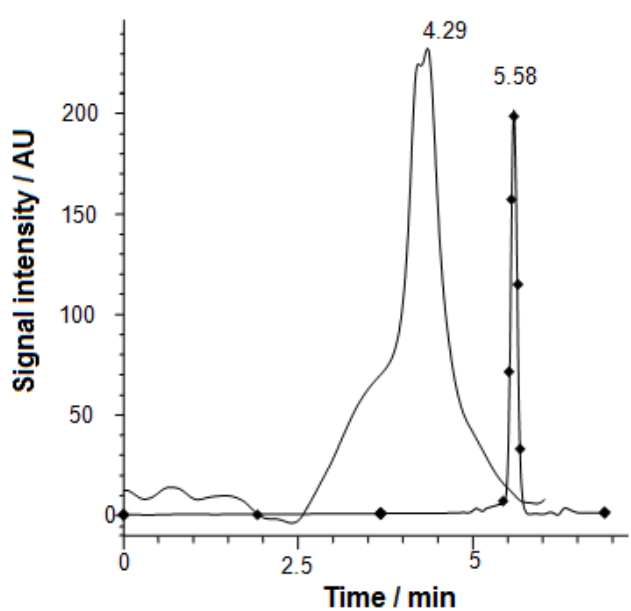

a)

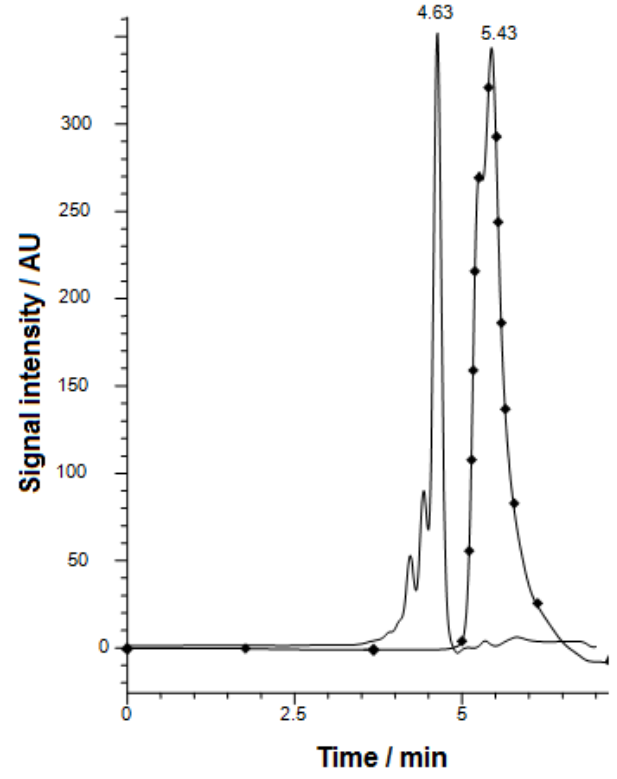

b)

Fig. 2 GPC curves of a) $\mathbf{1}$ - line ( $\bullet$ ) and I -line; b) 2-line( $\bullet$ ) and II- line

FT-IR spectrum of the metal complex shows a sharp band at $3240 \mathrm{~cm}^{-1}$ and a board one around 3444 $\mathrm{cm}^{-1}$ for symmetric and asymmetric NH stretching vibration (Fig. 3) [23]. Due to the reaction of the complex and the oxirane groups, NH bands in the spectrum of the polymer disappear with reaction progress. Band at $1640 \mathrm{~cm}^{-1}$ attributed to $\mathrm{vC}=\mathrm{O}$ in the spectrum of the polymer is missing, while band from $v \mathrm{C}=\mathrm{N}$ vibration is unchanged, confirming thus the proposed reaction scheme. The intensity of $\gamma \mathrm{C}-\mathrm{O}$ epoxy band at $915 \mathrm{~cm}^{-1}$ and the stretching of $\mathrm{C}-\mathrm{O}-\mathrm{C}$ (oxirane) at $830 \mathrm{~cm}^{-1}$ decrease as a result of the reaction. By opening the oxirane rings hydroxyl groups are formed. The broad band for $\mathrm{OH}$ stretching at $3512 \mathrm{~cm}^{-1}$ appearing in the spectrum of the polymer additionally confirms the opening of oxirane ring. 


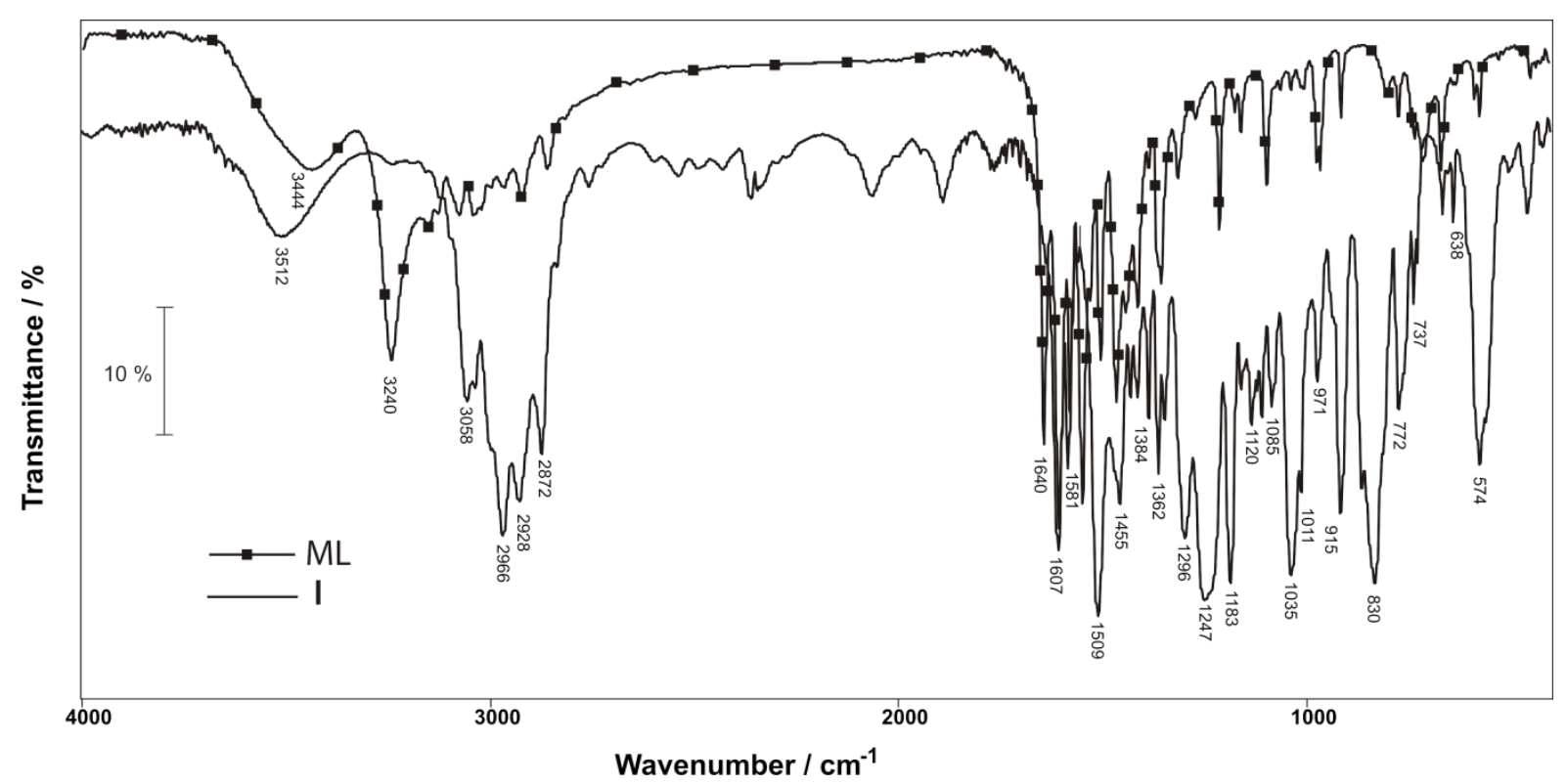

a)

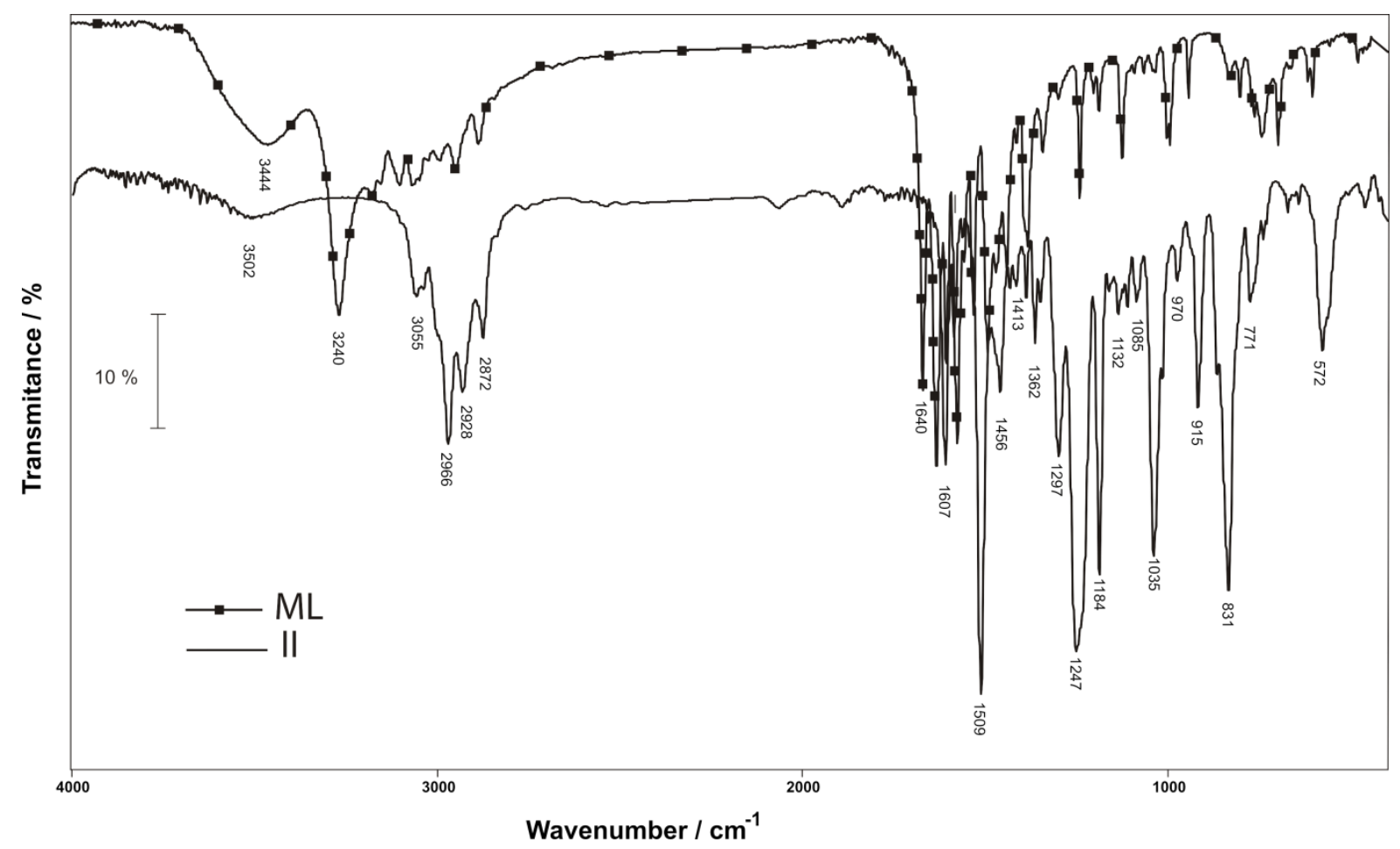

b)

Fig. 3 FT-IR spectrum of the ML complex and the: a) polymer I and b) polymer II

Thermal decomposition of hybrid polymers

The thermal stability and the decomposition of the hybrid materials were studied by TG/DSC and TG/DTA-MS measurements. Typical TG/DSC curve sets for the hybrid polymer I recorded in flowing nitrogen and air atmosphere are presented in Figure 4. As can be seen, the thermal stability of 
I is practically independent on the atmosphere. Also, the decomposition is exothermic in both atmospheres and show a similar course. As a consequence of the presence of oxygen the decomposition is more intensive in air and is accomplished above $550{ }^{\circ} \mathrm{C}$ without residue.

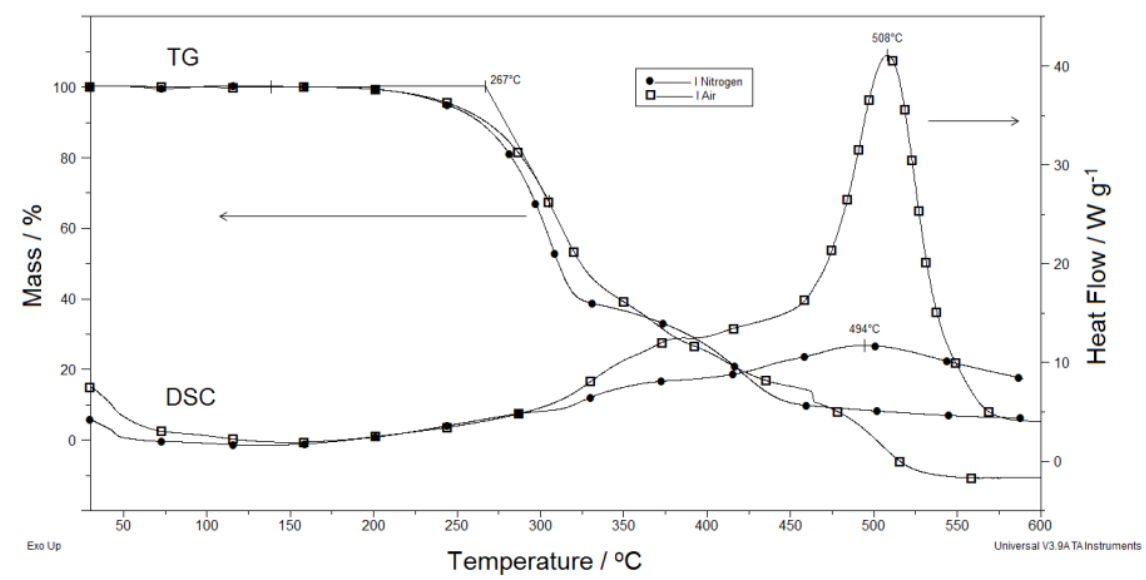

Fig. 4 Simultaneous TG/DSC curves for $\mathbf{I}$ in air and nitrogen

In Figure 5 the comparison of TG curves in flowing nitrogen for Araldite (sample 1) and its hybrid polymer I obtained in repeated synthesis are presented.

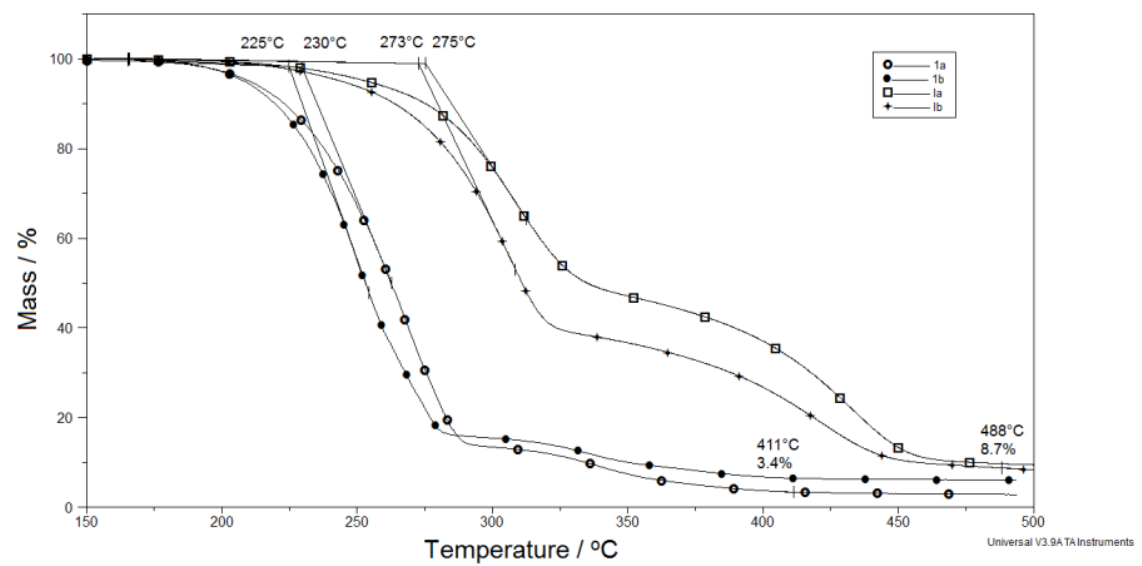

Fig. 5 TG curves for $\mathbf{1}$ and its hybrid polymer $\mathbf{I}$ with MLin nitrogen. Notations $\mathbf{a}$ and $\mathbf{b}$ stand for the same materials obtained in repeated syntheses.

The onset temperature for the thermal degradation of $\mathbf{1}$ is less by about $50{ }^{\circ} \mathrm{C}$ compared to that of $\mathbf{I}$. The decomposition patterns of both $\mathbf{1}$ and $\mathbf{I}$ are principally the same and consists of two main steps. However, the mass loss for $\mathbf{1}$ in the first step is significantly higher compared to the corresponding mass loss for I. This observation and the somewhat higher thermal stability of the new material support the reaction between the metal complex and epoxy monomer. The decomposition of $\mathbf{1}$ is accomplished around $400{ }^{\circ} \mathrm{C}$ with a tar residue of about $3 \%$. On the contrary, the decomposition of I ends at $\sim 500{ }^{\circ} \mathrm{C}$ and with a residue of $\sim 9 \%$ as a result of metal content of the sample. As the 
repeatability of the decomposition for pure $\mathbf{1}$ is not ideal, the relatively small differences in decomposition route of $\mathbf{I}$, obtained in two different syntheses, refer to an acceptable repeatability of the synthetic procedure.

The hybrid polymer II obtained by the reaction of ML with $\mathbf{2}$ has a somewhat lower stability than I and in nitrogen is practically stable to $250{ }^{\circ} \mathrm{C}$. Up to $200{ }^{\circ} \mathrm{C}$ the mass loss of about $3 \%$ is most probably due to the evaporation of xylene solvent trapped in the polymer matrix. The repeatability of the TG measurements is excellent (see Fig 6). However, as shown in Fig 6, the TG curves of II obtained in the repeated synthesis are less similar than those of the hybrid polymer $\mathbf{I}$. The reason for this might be related to the experimental difficulties to prepare a homogenous mixture of the reacting components and at the same time the significantly higher reactivity of the mixture.

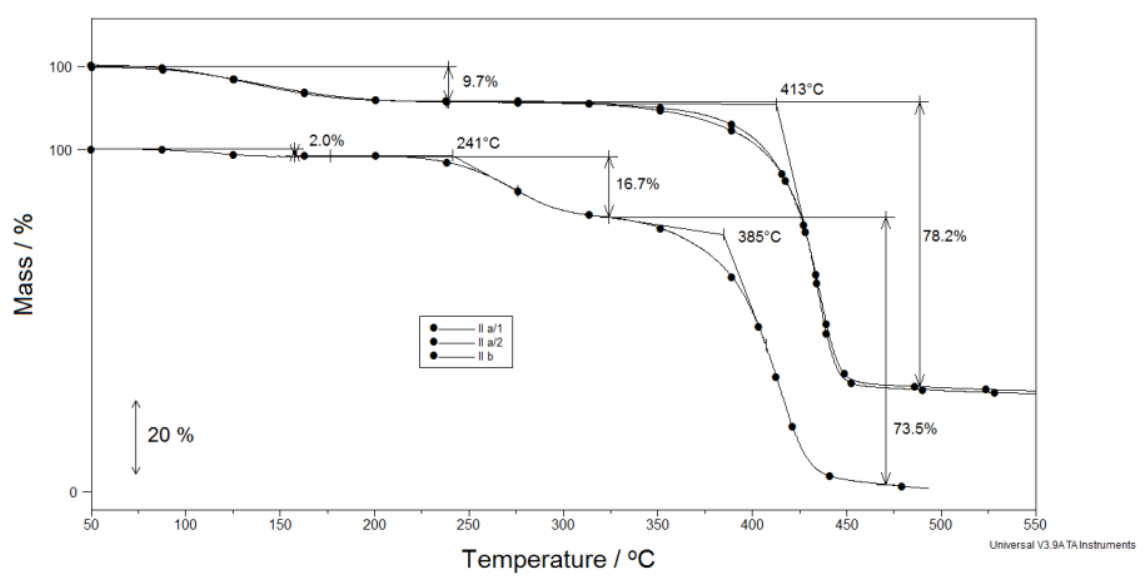

Fig. 6 TG curves of II in $\mathrm{N}_{2}$, recorded with samples obtained from repeated syntheses (a, b) and the repeatability of the measurement $(\mathrm{a} / 1$ and $\mathrm{a} / 2)$

In Fig 7 DTG curves for hybrid polymers in air and nitrogen are presented. The decomposition mechanism of $\mathbf{I}$ is only slightly affected by the atmosphere and is principally the same in both atmospheres. Due to highly exothermic decomposition/burning, samples in air decompose without residue at $500{ }^{\circ} \mathrm{C}$, the gas carrier carrying away particles of $\mathrm{CuO}$, too. Surprisingly, the thermal stability of $\mathbf{I}$ is somewhat higher in air (cc. $30{ }^{\circ} \mathrm{C}$ ) than in nitrogen. On the contrary, the thermal stability of II is significantly higher in nitrogen. Its decomposition in air takes place in several more or less separated steps (Fig 7), while in nitrogen it decomposes practically in one step with a residue of about $10 \%$. 


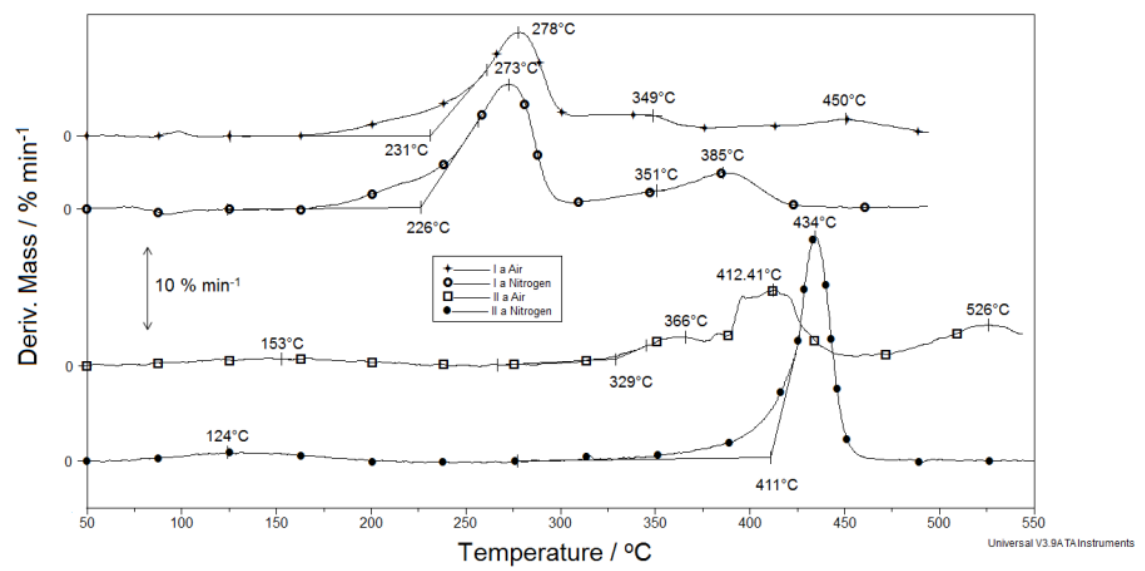

Fig 7 DTG curves for samples I and II in air and nitrogen

\section{The mechanism of decomposition}

To understand the reaction mechanism of the thermal decomposition, continuous monitoring of the departing gaseous products by means of hyphenated techniques like TG-FTIR [24] or TG-MS [25] is widely used. The thermal degradation products of some flame retardant epoxy resins were already studied [26-28]. In this paper the coupled TG-MS data were used not only for monitoring of the decomposition but to obtain additional proof about the reaction of the complex with epoxy resins and to prove the proposed reaction scheme for hybrid polymer formation. Fragments, related to the proposed structure are listed in Table 1.

Table 1 Assignment of the fragments

\begin{tabular}{lll}
\hline$m / z$ & Fragment & Fragment \\
\hline 41 & $\mathrm{C}_{3} \mathrm{H}_{5}^{+}$ & 91 \\
44 & $\mathrm{C}_{2} \mathrm{H}_{3} \mathrm{~N}^{+}$ & \\
50 & $\mathrm{CO}_{2}, \mathrm{C}_{2} \mathrm{H}_{4} \mathrm{O}^{+}$ & \\
56 & $\mathrm{C}_{4} \mathrm{H}_{2}^{+}$ & \\
$\mathrm{C}_{3} \mathrm{H}_{4} \mathrm{O}^{+}$ &
\end{tabular}

In low temperature region in II there is a mass loss of about $4 \%$ to $150{ }^{\circ} \mathrm{C}$. By coupled TG-MS measurements a strong signal with $m / z=91$ is detected, that is missing in the MS spectrum of all the other samples. As the starting material for the synthesis of II is a xylene solution of the epoxy resin $\mathbf{2}$, this peak is related to xylene [29] evaporation. In addition, traces of water were detected around 150 
${ }^{\circ} \mathrm{C}$ in both I and II. Due to the presence of the free $-\mathrm{OH}$ groups, formed in the polymerization reaction, peak for water elimination is detected at around $300{ }^{\circ} \mathrm{C}$ in I and at $440{ }^{\circ} \mathrm{C}$ in II.

The DTG curves and the corresponding MS curves with selected characteristic $\mathrm{m} / \mathrm{z}$ data for $\mathbf{1}, \mathbf{I}, \mathbf{I I}$ and ML are presented in Figs $8 \mathrm{a}-8 \mathrm{~d}$.

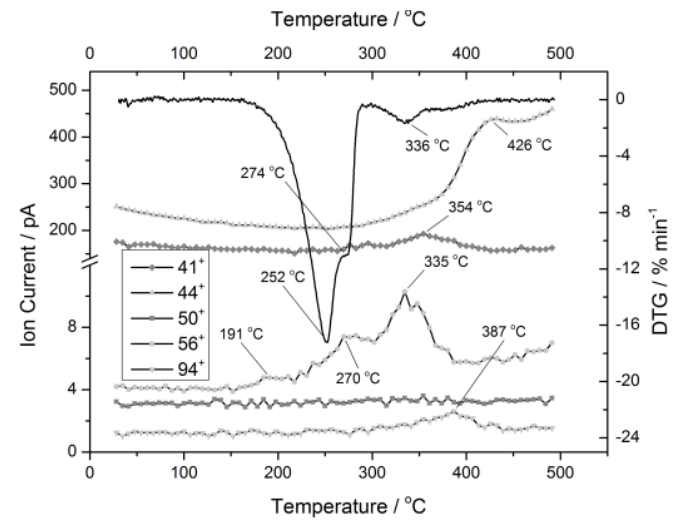

a) 1

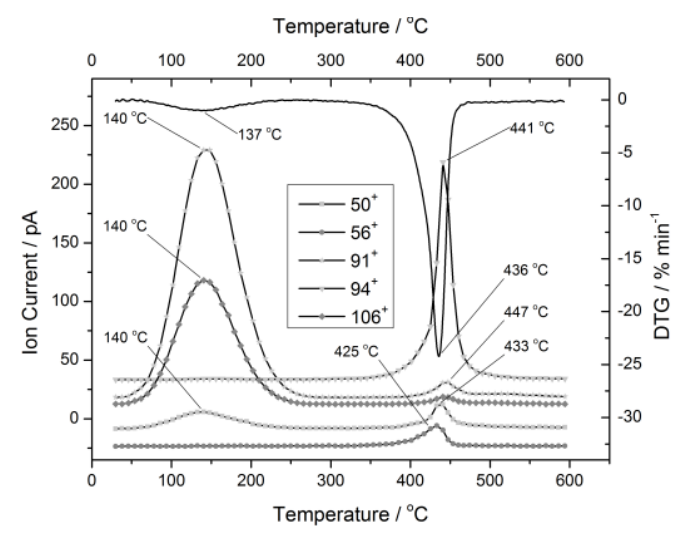

c) II

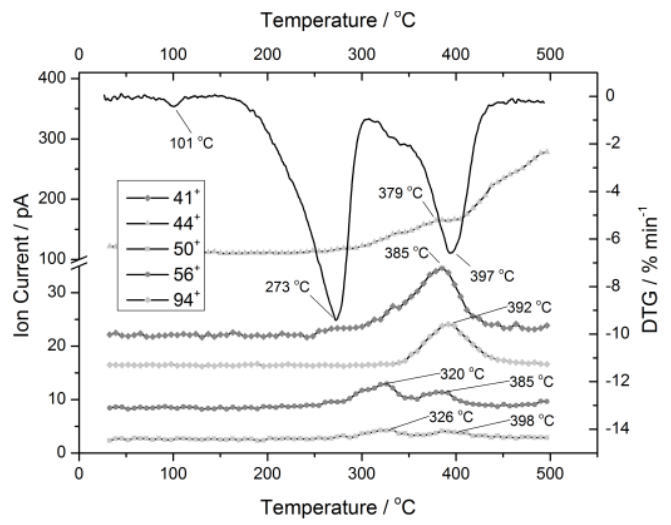

b) $\mathbf{I}$

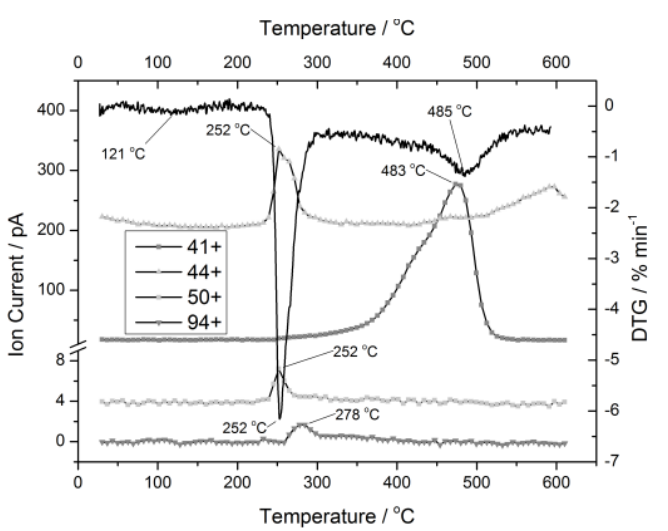

d) $M L$

Fig. 8 Ion currents for selected fragments with the corresponding DTG curves for 1, I, 2, II and ML. For better comparison the curves are shifted along the Ion Current axis

All the selected fragments in the $m / z$ range of $1-190$ can be found in the mass spectrum of Araldite [29]. However, the peaks intensities in different samples are very different, in part because of the thermal decomposition and also because of the different composition and structure of the new materials.

In accordance with the structure of bisphenol A diglycidyl ether (1), fragment with $\mathrm{m} / \mathrm{z}=41$ appearing above $300{ }^{\circ} \mathrm{C}$ most probably belongs to $\mathrm{CH}_{2}=\mathrm{CH}-\mathrm{CH}_{2}{ }^{+}$ion. In $\mathbf{I}$ its appearance refers thus to the break of the $\mathrm{O}-\mathrm{C}$ bond formed by oxirane group in the polymerization reaction. The intensity of this peak is however significantly lower in II that might be expected on the basis of the differences in the degree of cross-linking compared to $\mathbf{I}$. The same fragment during the decomposition of ML is 
detected at a significantly higher temperature $\left(t_{\text {peak }}=473{ }^{\circ} \mathrm{C}\right)$. In principle it can belong to allyl group, too, but could be formed also by decomposition of the pyrazole ring or metallacycles giving $\mathrm{CH}_{3}-$ $\mathrm{C} \equiv \mathrm{N}^{+}$. As this is the most intensive, asymmetric peak in the spectrum of ML, most probably both decomposition routes are feasible.

Fragment with $\mathrm{m} / \mathrm{z}=44$ is usually a result of $\mathrm{CO}_{2}$ formation and at the thermal decomposition of similar epoxy resins it is assigned to $\mathrm{CO}_{2}$ evolution [30]. It was found that the ion current curves during combustion and pyrolysis of an epoxy and phenol formaldehyde resins blend had identical shapes, only the peak intensity was significantly lower in helium, suggesting a decomposition mechanism independent on the atmosphere [30]. However, fragment $\mathrm{C}_{2} \mathrm{H}_{4} \mathrm{O}^{+}$has the same $\mathrm{m} / \mathrm{z}$ ratio, and may be formed by splitting of the bond formed by oxirane group, too. In principle $\mathrm{CO}_{2}{ }^{+}$and $\mathrm{C}_{2} \mathrm{H}_{4} \mathrm{O}^{+}$could be differentiated using the corresponding daughter ions. However their intensity is too small and is near the range of the noise intensity. On the basis of the similar DTG curves of $\mathbf{I}$ in nitrogen and air (Fig 7) one would expect a similar decomposition mechanism. However, the comparison of the ion current curves for $m / z=44$ refer to a different decomposition scheme of $\mathbf{I}$ in air and nitrogen. As can be seen in Fig 9, the formation of a fragment with $m / z=44$ in nitrogen can be neglected. On the contrary, the ion current of $\mathrm{CO}_{2}$ in $\mathbf{1}$ measured in nitrogen is lower but has a similar shape as $\mathbf{I}$ in air. This observation suggests that the presence of metal ion alters the decomposition route of $\mathbf{I}$ in inert atmosphere of $\mathrm{N}_{2}$.

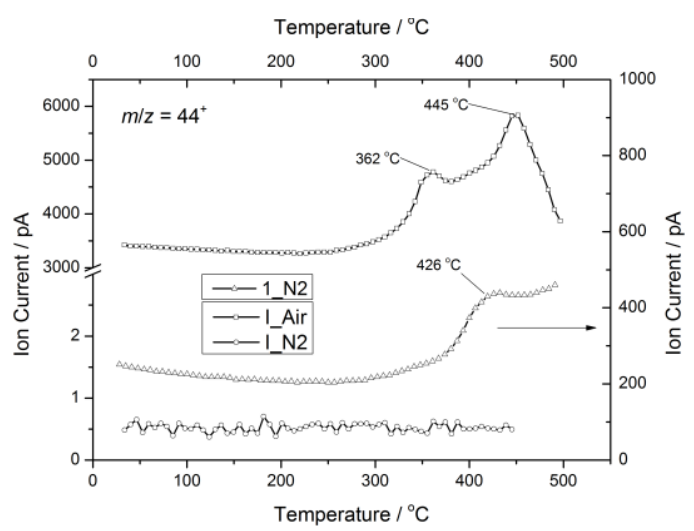

Fig. 9 Ion current for $m / z=44$ in I recorded in air and nitrogen compared with the corresponding ion current in $\mathbf{1}$ in nitrogen

In ML, beside $\mathrm{CO}_{2}$ evolution, $\mathrm{m} / \mathrm{z}=44$ can be the result of the loss of the acetyl substituent of the pyrazole ring, i.e. the $\mathrm{CH}_{3}-\mathrm{CH}=\mathrm{O}^{+}$group. Unfortunately, we could not find a peak characteristic for ML only as the fragments evolving during the thermal decomposition appear practically in all tested materials. The fragment with $\mathrm{m} / \mathrm{z}=50$ in the spectrum of Araldite (1) is present with a relative intensity of less than $1 \%$ [29]. During the thermal decomposition of 1 the ion current for $m / z=50$ is very low and is constant in the observed temperature range (see Fig 8 a). On the contrary, among the 
fragments appearing in the decomposition of ML there is a peak with $\mathrm{m} / z=50$ at the relative intensity higher than $10 \%$ (Fig $8 \mathrm{~d}$ ). The composition of the fragment corresponds to $\mathrm{C}_{4} \mathrm{H}_{2}{ }^{+}$that can be easily formed from the 4-acetyl-5-methylpyrazole moiety. As the intensity of this peak in epoxy resins is extremely low, its appearance in the spectra of $\mathbf{I}$ and $\mathbf{I I}$ would prove the chemical reaction of ML with the epoxy resins.

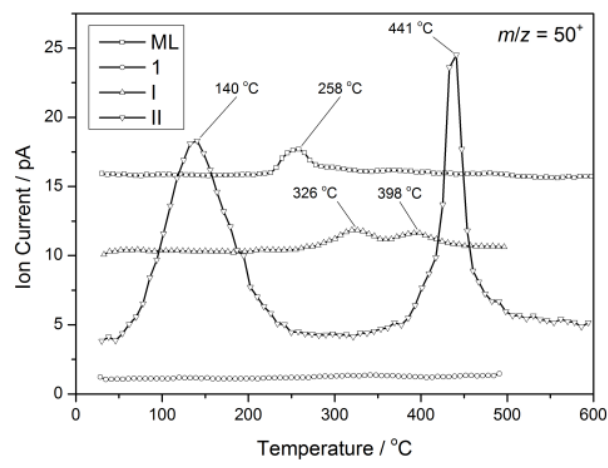

Fig. 10 Ion current for $m / z=50$ in $\mathbf{1}, \mathbf{I}$, II and $\mathbf{M L}$

Indeed, in Fig 10 the differences in ion current of $m / z=50$ in $\mathbf{1}, \mathbf{I}, \mathbf{I I}$ and ML support the proposition of the chemical reaction between the resins and ML. The $\mathrm{m} / \mathrm{z}=50$ current intensity in ML has a maximum at $250{ }^{\circ} \mathrm{C}$, while in $\mathbf{1}$ it is significantly less and does not change with temperature. In $\mathbf{I}$ the temperature of the current maximum is shifted from $250{ }^{\circ} \mathrm{C}$ to $330{ }^{\circ} \mathrm{C}$ and $390{ }^{\circ} \mathrm{C}$. The significantly higher peak temperatures in I compared to its peak temperature in ML support the chemical bonding of the complex. The appearance of double MS peaks (Fig 10) in I refers to different building units in the polymer, in accordance with multiple peaks in the corresponding GPC curve (Fig 2) and a high polydispersity index for a wider molecular mass distributions. As II is prepared using a xylene solution of the epoxy resin, the characteristic signals for xylene fragmentation [29] with $\mathrm{m} / \mathrm{z}$ of $91-$ $106-50$ and relative intensity ratio of $1 / 0.5 / 0.08$ appear at $140{ }^{\circ} \mathrm{C}$. The same peaks were detected also at $440{ }^{\circ} \mathrm{C}$. However, the corresponding relative intensity ratio has been changed in favour of $\mathrm{m} / z=50$, being the most intensive among the $m / z=91-106-50$ peak set now with an intensity ratio of 0.63/0.33/1 (Fig. 8 c). In addition, while the low temperature peaks positions are identical, at high temperatures the position of the peaks is somewhat shifted to each other. The high temperature of the appearance, the different shapes and peak temperatures and the substantial change in their relative intensity ratio confirm the presence of ML as a building unit of II. However, the presence of all three peaks also refers to the trace of xylene trapped in the matrix.

During the thermal decomposition peaks with $\mathrm{m} / z=56$ and $\mathrm{m} / \mathrm{z}=57$ appear in $\mathbf{1}$, I and II, but in ML their intensities are negligible. The peak intensities for both fragments are in the same range, the peak with $m / z=56$ being more intensive, therefore it is presented in Figs 8 a-c. In the mass spectrum of 
Araldite [29] the most intensive peak in the range below $200 \mathrm{amu}$ belongs to fragment with $\mathrm{m} / z=57$ and most probably involves the splitting of oxirane group. Peak with $\mathrm{m} / z=56$ is consistent with a fragment of $\mathrm{C}_{3} \mathrm{H}_{4} \mathrm{O}^{+}$that can be formed by breaking of the bond between the phenoxy and oxirane groups.

Fragment with $m / z=94$ has been found also in all compounds. Regarding the different composition of $\mathbf{1}, \mathbf{I}, \mathbf{I I}$ and ML, its origin is a result of different reactions. In the epoxy containing unit it has been detected in different resins [25, 30, 31] and is assigned to ionized phenol. In ML the corresponding fragment may be consistent with $\mathrm{C}_{5} \mathrm{H}_{6} \mathrm{~N}_{2}^{+}$that could be formed from the pyrazole moiety, but as well from the metallacycle fragmentation.

Peak with $\mathrm{m} / z=106$ belongs to xylene fragmentation only, as is present only in II with significant intensity. By isothermal heating xylene can be removed below $100{ }^{\circ} \mathrm{C}$, but its traces still were found during the decomposition of II above $400{ }^{\circ} \mathrm{C}$.

\section{Conclusions}

Novel epoxy polymers have been obtained by curing the epoxy resins, based on diglycidyl ether of bisphenol $\mathrm{A}$ and $\left[\mathrm{Cu}(\operatorname{ampf}) \mathrm{Cl}_{2}\right]$, ampf $=N, N$ '-bis(4-acetyl-5-methylpyrazole-3-yl)formamidine. The hybrid polymer synthesized with Araldite has higher molecular masses, $25.000 \mathrm{~g} \mathrm{~mol}^{-1}$ and broader molecular mass distribution compared with a polymer based on CHS epoxy with molecular mass of $3.200 \mathrm{~g} \mathrm{~mol}^{-1}$. The reaction between the epoxy polymers and the metal complex as cross-linker was followed by means of FT-IR spectroscopy and DSC runs. The disappearance of $\mathrm{NH}$ and $\nu \mathrm{C}=\mathrm{O}$ bands in the spectrum of the complex and the decreasing intensity of $\gamma \mathrm{C}-\mathrm{O}$ epoxy and $\mathrm{C}-\mathrm{O}-\mathrm{C}$ (oxirane) bands in the IR spectrum of the polymers with unchanged $v \mathrm{C}=\mathrm{N}$ vibration support the proposed reaction scheme. Exothermic peaks in the $100-200{ }^{\circ} \mathrm{C}$ temperature range and the values of the reaction enthalpies ( $\left.>100 \mathrm{~J} \mathrm{~g}^{-1}\right)$ suggest a high reactivity between the components.

The formation of chemical bonds between the epoxy resins and the metal complex was additionally supported by the results of coupled TG-MS measurements. The hybrid materials show an increased thermal stability compared with that of the starting compounds. Their low conductivities in tetrahydrofuran confirm the non-ionic structure of the new polymers in solution. Bue to the presence of copper(II) it is supposed that the new materials in solution show electroactivity. Therefore the electrochemical properties of the novel hybrid materials are under study.

\section{ACKNOWLEDGEMENTS}

This paper was supported by the Ministry of Education, Science and Technological Development, Republic of Serbia; contract grant numbers: III 45022 and ON 172014. B. Barta Holló acknowledges a Domus Hungarica Fellowship. I. M. Szilágyi acknowledges a János Bolyai Research Fellowship of the Hungarian Academy of Sciences, and an OTKA-PD-109129 grant. 


\section{Literature}

1. Ellis B. Chemistry and Technology of Epoxy Resins, Blackie Academic and Professional, Glasgow (1993) p. 37.

2. Cella J, Schwabacher A, Shuls A. Cationic Chelate Salts of Main Group Elements as Curing Agents for Epoxy Resins. Znd Eng Chem Prod Res Deu. 1983;22:20-25.

3. Kurnoskin AV, Lapitsky VA. Sinteza Struktura Svoistva Medsoderzhashchikh Polimerov, Abstracts of Papers. Vsesoyuznaya Konferentsiya, Zvenigorod, (1988) 55.

4. Kurnoskin AV. Metal-containing epoxy polymers. In:Cheremisinoff NP, Cheremisinoff PN, editors. Handbook of applied polymer processing technology. New York:Marcel Dekker, (1996) 703-65.

5. Kurnoskin AV. Metalliferous epoxy-chelate polymers:analytical review. JMS Rev. Macromol. Chem. Phys. 1996;C36:457-599.

6. Kurnoskin AV. Epoxy polymer modification with metals. Polym. Comp. 1993;14:481-90.

7. Lin KF, Shu WY, Wey TL. Organotransition-metal complexes as additives for epoxy resins: Part 1. Their effects on toughness and morphology of epoxy resins. Polymer 1993;34:277-88.

8. Lin KF, Wang WH. Imidazole and chromium acetylacetonate as additives for the cure and fracture-toughness of epoxy resins and their composites. Polym. Comp. 1995;16:269-75.

9. Lin KF, Shu WY, Wey TL. Organotransition-metal complexes as additives for epoxy resins: Part 2. Interaction with epoxy resins. Polymer 1993;34:2162-68.

10. Anand M, Srivastava AK. Synthesis and characterization of epoxy resins containing arsenic acrylate. Polym. Eng. Sci. 1997;37:183-7.

11. Anand M, Srivastava AK. Synthesis and characterization of epoxy resins containing chromium acrylate. Angew. Makromol. Chem. 1994;219:1-10.

12. Anand M, Srivastava AK, Synthesis of zinc-containing epoxy resin. J. Appl. Polym. Sci. 1994;51:203-11.

13. Kurnoskin AV, Korshikov LA, Kanovich MZ, Filatova LD, Soloveva EK, Optimization of the curing regime for epoxychelate composite materials with the results of evaluation of residual stresses, Fibre. Chem. 1995;27:266-9.

14. Chantarasiria N, Tuntulania T, Tongraunga P, Seangprasertkit-Mageea R, Wannarong W. New metal-containing epoxy polymers from diglycidyl ether of bisphenol A and tetradentate Schiff base metal complexes. Eur. Polym. J. 2000;36:695-702.

15. Kurnoskin AV, Kanovitch MZ, Ilyin VM, Gusev LL, Bekeshko VV. Application of epoxychelate composites in automobile industry. Inf. Bull. Khim. Prom. SEV 1990;6:25-9.

16. Hamerton I, Howlin BJ, Jepson P. Metals and coordination compounds as modifiers for epoxy resins, Coordin. Chem. Rev. 2002;224:67-85. 
17. Kurnoskin AV. Epoxy Chelate Copper-Containing Polymers: Their Chemistry and Production, Polym. Plas.Tech. Eng. 1992;31:505-25.

18. Kurnoskin AV. Metalliferous epoxy chelate polymers: 1. Synthesis and properties, Polymer 34 (1993) 1060-1067.

19. Kurnoskin AV. Epoxy polymer modification with metals, Polym. Comp. 1993;14:481-90.

20. Holló B, Leovac VM, Bombicz P, Kovács A, Jovanović LS, Bogdanović G, Kojić V, Divjaković V, Joksović MD, Mészáros Szécsényi K. Synthesis, Structural, DFT, and Cytotoxicity Studies of $\mathrm{Cu}^{\mathrm{II}}$ and $\mathrm{Ni}^{\mathrm{II}}$ Complexes with 3-Aminopyrazole Derivatives, Aust. J. Chem. 2010;63:1557-64.

21. Tatsumiya S, Yokokawa K, Miki K. A dynamic DSC study of the curing process of epoxy resin, J. Therm. Anal. 1997;49:123-129.

22. Rosu D, Cascaval C, Mustata F, Ciobanu C, Cure kinetics of epoxy resins studied by nonisothermal DSC data, Thermochimi. Acta. 2002;383:119-127.

23. Silverstein R, Bassler C, Morrill T. Spectrometric identification of organic compounds, Wiley International Edition, New York, third edition, (1974) 108.

24. Quan C, Li A, Gao N. Research on pyrolysis of PCB waste with TG-FTIR and Py-GC/MS, J. Therm. Anal. Calorim. 2012;110:1463-70.

25. Xu J, He Z, Wu W, Ma H, Xie J, Qu H, Jiao Y. Study of thermal properties of flame retardant epoxy resin treated with hexakis[p-(hydroxymethyl)phenoxy]cyclotriphosphazene, J. Therm. Anal. Calorim. 2013;114:1341-50.

26. Balabanovicha AI, Hornunga A, Merza D, Seifert H. The effect of a curing agent on the thermal degradation of fire retardant brominated epoxy resins, Polym. Degrad. Stab. 2004;85:713-23.

27. Wang X, Hu Y, Song L, Xing W, Lu H. Thermal degradation mechanism of flame retarded epoxy resins with a DOPO-substitued organophosphorus oligomer by TG-FTIR and DP-MS, J. Anal. App. Pyrol. 2011;92:164-70.

28. Qian L, Ye L, Qiu Y, Qu S. Thermal degradation behavior of the compound containing phosphaphenanthrene and phosphazene groups and its flame retardant mechanism on epoxy resin, Polymer 2011;52:5486-93.

29. http://webbook.nist.gov/ last accessed 12.12.2013.

30. Ahamad T, Alshehri SM. Thermal degradation and evolved gas analysis of epoxy (DGEBA)/novolac resin blends (ENB) during pyrolysis and combustion, J. Therm. Anal. Calorim. 2013;111:445-51.

31. Huang Z, Shi W. Thermal behavior and degradation mechanism of poly(bisphenyl acryloxyethyl phosphate) as a UV curable flame-retardant oligomer, Polym. Degrad. Stabil. 2006;91:1674-84. 